\title{
Spectral gap global solutions for degenerate Kirchhoff equations
}

\author{
Marina Ghisi \\ Università degli Studi di Pisa \\ Dipartimento di Matematica "Leonida Tonelli" \\ PISA (Italy) \\ e-mail: ghisi@dm.unipi.it \\ Massimo Gobbino \\ Università degli Studi di Pisa \\ Dipartimento di Matematica Applicata "Ulisse Dini" \\ PISA (Italy) \\ e-mail: m.gobbino@dma.unipi.it
}




\begin{abstract}
We consider the second order Cauchy problem

$$
u^{\prime \prime}+m\left(\left|A^{1 / 2} u\right|^{2}\right) A u=0, \quad u(0)=u_{0}, \quad u^{\prime}(0)=u_{1},
$$

where $m:[0,+\infty) \rightarrow[0,+\infty)$ is a continuous function, and $A$ is a self-adjoint nonnegative operator with dense domain on a Hilbert space.

It is well known that this problem admits local-in-time solutions provided that $u_{0}$ and $u_{1}$ are regular enough, depending on the continuity modulus of $m$, and on the strict/weak hyperbolicity of the equation.

We prove that for such initial data $\left(u_{0}, u_{1}\right)$ there exist two pairs of initial data $\left(\bar{u}_{0}, \bar{u}_{1}\right)$, $\left(\widehat{u}_{0}, \widehat{u}_{1}\right)$ for which the solution is global, and such that $u_{0}=\bar{u}_{0}+\widehat{u}_{0}, u_{1}=\bar{u}_{1}+\widehat{u}_{1}$.

This is a byproduct of a global existence result for initial data with a suitable spectral gap, which extends previous results obtained in the strictly hyperbolic case with a smooth nonlinearity $m$.
\end{abstract}

Mathematics Subject Classification 2000 (MSC2000): 35L70, 35L80, 35L90.

Key words: uniqueness, integro-differential hyperbolic equation, degenerate hyperbolic equation, continuity modulus, Kirchhoff equations, Gevrey spaces. 


\section{Introduction}

Let $H$ be a real Hilbert space. For every $x$ and $y$ in $H$, let $|x|$ denote the norm of $x$, and let $\langle x, y\rangle$ denote the scalar product of $x$ and $y$. Let $A$ be an unbounded linear operator on $H$ with dense domain $D(A)$. We always assume that $A$ is self-adjoint and nonnegative, so that for every $\alpha \geq 0$ the power $A^{\alpha}$ is defined in a suitable domain $D\left(A^{\alpha}\right)$.

Given a continuous function $m:[0,+\infty) \rightarrow[0,+\infty)$ we consider the Cauchy problem

$$
\begin{gathered}
u^{\prime \prime}(t)+m\left(\left|A^{1 / 2} u(t)\right|^{2}\right) A u(t)=0, \quad \forall t \in[0, T), \\
u(0)=u_{0}, \quad u^{\prime}(0)=u_{1} .
\end{gathered}
$$

It is well known that (1.1), (1.2) is the abstract setting of the Cauchy-boundary value problem for the quasilinear hyperbolic integro-differential partial differential equation

$$
u_{t t}(t, x)-m\left(\int_{\Omega}|\nabla u(t, x)|^{2} d x\right) \Delta u(t, x)=0 \quad \forall(x, t) \in \Omega \times[0, T),
$$

where $\Omega \subseteq \mathbb{R}^{n}$ is an open set, and $\nabla u$ and $\Delta u$ denote the gradient and the Laplacian of $u$ with respect to the space variables.

Equation (1.1) is called strictly hyperbolic if

$$
m(\sigma) \geq \nu>0 \quad \forall \sigma \geq 0
$$

Equation (1.1) is called weakly (or degenerate) hyperbolic if

$$
m(\sigma) \geq 0 \quad \forall \sigma \geq 0 .
$$

Existence of local/global solutions to (1.1), (1.2) has long been investigated in the last century. The theory is well established in the case of local solutions, which are known to exist in the following situations.

(L1) When equation (1.1) is strictly hyperbolic, $m$ is Lipschitz continuous, and initial data $\left(u_{0}, u_{1}\right) \in D\left(A^{3 / 4}\right) \times D\left(A^{1 / 4}\right)$ (see [1] and the references quoted therein).

(L2) When equation (1.1) is weakly hyperbolic, $m$ is continuous, and initial data are analytic. In this case solutions are actually global (see [2], [5], [6]).

(L3) More generally, when initial data belong to suitable intermediate spaces, depending on the continuity modulus of $m$, and on the strict/weak hyperbolicity of (1.1) (see [11] and [8]). This is a sort of interpolation between (L1) and (L2). We refer to section 2 for precise definitions of the functional spaces in the abstract framework and a formal local existence statement (Theorem A). 
Existence of global solutions is a much more difficult problem, and it is still widely open. A positive answer has been given in the case (L2), and in some special situations: quasi-analytic initial data (see [15]), or Sobolev-type data but special nonlinearities $m$ (see [16]), or dispersive operators and small data (see [10], [7]). But for (L2) all these results assume the strict hyperbolicity and the Lipschitz continuity of $m$.

Recently R. MANFRIN [13, 14] (see also [12]) considered once again the strictly hyperbolic case with a smooth nonlinearity. He proved global existence in a special class of nonanalytic initial data. Manfrin's spaces are not vector spaces and do not contain any Gevrey space $\mathcal{G}_{s}$ with $s>1$. However they have the following astonishing property:

(M) every pair of initial conditions $\left(u_{0}, u_{1}\right) \in D(A) \times D\left(A^{1 / 2}\right)$ is the sum of two pairs of initial conditions in Manfrin's spaces, i.e., the sum of two initial conditions for which the solution is global!

This theory requires the strict hyperbolicity and some smoothness of $m$, which is assumed to be of class $C^{2}$ both in [13] and [14].

In this paper we extend Manfrin's theory to the general situation of (L3). We consider indeed both the strictly hyperbolic and the weakly hyperbolic case, and a nonlinearity $m$ with a given continuity modulus. In Theorem 3.1 we prove global existence for initial data in a suitable subset of the spaces involved in (L3). In analogy with Manfrin's spaces, the definition (3.1) of our subset is made in terms of the spectral resolution of initial data. Of course our subset is not a vector space and it doesn't even contain all analytic functions. Nevertheless in Proposition 3.2 we show that this subset satisfies property (M) in the spaces involved in (L3).

From the point of view of property (M) our result extends Manfrin's one also in the framework (L1). In this case we obtain indeed property (M) for initial data in $D\left(A^{3 / 4}\right) \times D\left(A^{1 / 4}\right)$ and a locally Lipschitz continuous nonlinearity $m$, instead of initial data in $D(A) \times D\left(A^{1 / 2}\right)$ and $m \in C^{2}$.

This paper is organized as follows. In section 2 we recall the definition of continuity modulus and Gevrey-type functional spaces, and we state the local existence result for the case (L3). In section 3 we introduce our spaces and we state our main results. In section 4 we prove these results.

\section{Preliminaries}

For the sake of simplicity we assume that $H$ admits a countable complete orthonormal system $\left\{e_{k}\right\}_{k \geq 1}$ made by eigenvectors of $A$. We denote the corresponding eigenvalues by $\lambda_{k}^{2}\left(\right.$ with $\left.\lambda_{k} \geq 0\right)$, so that $A e_{k}=\lambda_{k}^{2} e_{k}$ for every $k \geq 1$.

Under this assumption we can work with Fourier series. However, any definition or statement of this section can be easily extended to the general setting just by using the 
spectral decomposition instead of Fourier series. The interested reader is referred to [1] for further details.

By means of the orthonormal system every $u \in H$ can be written in a unique way in the form $u=\sum_{k=1}^{\infty} u_{k} e_{k}$, where $u_{k}=\left\langle u, e_{k}\right\rangle$ are the Fourier components of $u$. With these notations for every $\alpha \geq 0$ we have that

$$
D\left(A^{\alpha}\right):=\left\{u \in H: \sum_{k=1}^{\infty} \lambda_{k}^{4 \alpha} u_{k}^{2}<+\infty\right\} .
$$

Let now $\varphi:[0,+\infty) \rightarrow(0,+\infty)$ be any function. Then for every $\alpha \geq 0$ and $r>0$ one can set

$$
\|u\|_{\varphi, r, \alpha}^{2}:=\sum_{k=1}^{\infty} \lambda_{k}^{4 \alpha} u_{k}^{2} \exp \left(r \varphi\left(\lambda_{k}\right)\right),
$$

and then define the spaces

$$
\mathcal{G}_{\varphi, r, \alpha}(A):=\left\{u \in H:\|u\|_{\varphi, r, \alpha}^{2}<+\infty\right\} .
$$

These spaces are a generalization of the usual spaces of Sobolev, Gevrey or analytic functions. They are Hilbert spaces with norm $\left(|u|^{2}+\|u\|_{\varphi, r, \alpha}^{2}\right)^{1 / 2}$. We also set

$$
\mathcal{G}_{\varphi, \infty, \alpha}(A):=\bigcap_{r>0} \mathcal{G}_{\varphi, r, \alpha}(A) .
$$

A continuity modulus is a continuous increasing function $\omega:[0,+\infty) \rightarrow[0,+\infty)$ such that $\omega(0)=0$, and $\omega(a+b) \leq \omega(a)+\omega(b)$ for every $a \geq 0$ and $b \geq 0$.

The function $m$ is said to be $\omega$-continuous if there exists a constant $L \in \mathbb{R}$ such that

$$
|m(a)-m(b)| \leq L \omega(|a-b|) \quad \forall a \geq 0, \forall b \geq 0 .
$$

The following result sums up the state of the art concerning existence of local solutions. We refer to Theorem 2.1 and Theorem 2.2 in [11] for the existence part, to [8] for some counterexamples, and to [9] for uniqueness issues.

Theorem A Let $\omega$ be a continuity modulus, let $m:[0,+\infty) \rightarrow[0,+\infty)$ be a (locally) $\omega$-continuous function, and let $\varphi:[0,+\infty) \rightarrow(0,+\infty)$.

Let us assume that there exists a constant $\Lambda$ such that

$$
\sigma \omega\left(\frac{1}{\sigma}\right) \leq \Lambda \varphi(\sigma) \quad \forall \sigma>0
$$

in the strictly hyperbolic case, and

$$
\sigma \leq \Lambda \varphi\left(\frac{\sigma}{\sqrt{\omega(1 / \sigma)}}\right) \quad \forall \sigma>0
$$


in the weakly hyperbolic case.

Let

$$
\left(u_{0}, u_{1}\right) \in \mathcal{G}_{\varphi, r_{0}, 3 / 4}(A) \times \mathcal{G}_{\varphi, r_{0}, 1 / 4}(A)
$$

for some $r_{0}>0$.

Then there exists $T>0$, and a nonincreasing function $r:[0, T] \rightarrow\left(0, r_{0}\right]$ such that problem (1.1), (1.2) admits at least one local solution

$$
u \in C^{1}\left([0, T] ; \mathcal{G}_{\varphi, r(t), 1 / 4}(A)\right) \cap C^{0}\left([0, T] ; \mathcal{G}_{\varphi, r(t), 3 / 4}(A)\right) .
$$

\section{Main result}

Let $\mathcal{L}$ denote the set of all sequences $\left\{\rho_{n}\right\}$ of positive real numbers such that $\rho_{n} \rightarrow+\infty$ as $n \rightarrow+\infty$. Given $\varphi:[0,+\infty) \rightarrow(0,+\infty),\left\{\rho_{n}\right\} \in \mathcal{L}, \alpha \geq 0$, and $\beta \geq 0$ we set

$$
\mathcal{G M}_{\varphi,\left\{\rho_{n}\right\}, \alpha}^{(\beta)}(A):=\left\{u \in H: \sum_{\lambda_{k}>\rho_{n}} \lambda_{k}^{4 \alpha} u_{k}^{2} \exp \left(\rho_{n}^{\beta} \varphi\left(\lambda_{k}\right)\right) \leq \rho_{n} \quad \forall n \in \mathbb{N}\right\}
$$

and then

$$
\mathcal{G} \mathcal{M}_{\varphi, \alpha}^{(\beta)}(A):=\bigcup_{\left\{\rho_{n}\right\} \in \mathcal{L}} \mathcal{G} \mathcal{M}_{\varphi,\left\{\rho_{n}\right\}, \alpha}^{(\beta)}(A) .
$$

These spaces are a generalization of Manfrin's spaces.

The following global existence result is the main result of this paper.

Theorem 3.1 Let $\omega$ be a continuity modulus, let $m:[0,+\infty) \rightarrow[0,+\infty)$ be a function satisfying (2.2), let $\varphi:[0,+\infty) \rightarrow(0,+\infty)$, and let $\left\{\rho_{n}\right\} \in \mathcal{L}$.

Let us assume that

- in the strictly hyperbolic case (2.3) holds true for a suitable $\Lambda$, and

$$
\left(u_{0}, u_{1}\right) \in \mathcal{G} \mathcal{M}_{\varphi,\left\{\rho_{n}\right\}, 3 / 4}^{(2)}(A) \times \mathcal{G} \mathcal{M}_{\varphi,\left\{\rho_{n}\right\}, 1 / 4}^{(2)}(A),
$$

- in the weakly hyperbolic case (2.4) holds true for a suitable $\Lambda$, and

$$
\left(u_{0}, u_{1}\right) \in \mathcal{G M}_{\varphi,\left\{\rho_{n}\right\}, 3 / 4}^{(3)}(A) \times \mathcal{G} \mathcal{M}_{\varphi,\left\{\rho_{n}\right\}, 1 / 4}^{(3)}(A) .
$$

Then problem (1.1), (1.2) admits at least one global solution u(t) with

$$
u \in C^{1}\left([0,+\infty) ; \mathcal{G}_{\varphi, r, 3 / 4}(A)\right) \cap C^{0}\left([0,+\infty) ; \mathcal{G}_{\varphi, r, 1 / 4}(A)\right)
$$

for every $r>0$. 
We conclude by speculating on these spaces. First of all it is easy to prove that

$$
\mathcal{G M}_{\varphi, \alpha}^{(\beta)}(A) \subseteq \mathcal{G}_{\varphi, \infty, \alpha}(A)
$$

for every admissible values of the parameters. On one hand this inclusion is "very strict". Roughly speaking indeed the inequalities in definition (3.1) require that the spectrum of $u$ "has a big hole after each $\rho_{n}$ ". For this heuristic reason we used "spectral gap solutions" to denote the solutions produced by Theorem 3.1.

On the other hand inclusion (3.5) is "not so strict" in the sense that

$$
\mathcal{G M}_{\varphi, \alpha}^{(\beta)}(A)+\mathcal{G} \mathcal{M}_{\varphi, \alpha}^{(\beta)}(A)=\mathcal{G}_{\varphi, \infty, \alpha}(A)
$$

for any admissible values of the parameters. We state this property more precisely in the case of pairs of initial data.

Proposition 3.2 Let $\varphi:[0,+\infty) \rightarrow(0,+\infty)$, and let

$$
\left(u_{0}, u_{1}\right) \in \mathcal{G}_{\varphi, \infty, 3 / 4}(A) \times \mathcal{G}_{\varphi, \infty, 1 / 4}(A) .
$$

Then for every $\beta \geq 0$ there exist $\left\{\bar{\rho}_{n}\right\}$ and $\left\{\widehat{\rho}_{n}\right\}$ in $\mathcal{L}$, and

$$
\begin{aligned}
& \left(\bar{u}_{0}, \bar{u}_{1}\right) \in \mathcal{G} \mathcal{M}_{\varphi,\left\{\bar{p}_{n}\right\}, 3 / 4}^{(\beta)}(A) \times \mathcal{G} \mathcal{M}_{\varphi,\left\{\bar{\rho}_{n}\right\}, 1 / 4}^{(\beta)}(A), \\
& \left(\widehat{u}_{0}, \widehat{u}_{1}\right) \in \mathcal{G M}_{\varphi,\left\{\widehat{p}_{n}\right\}, 3 / 4}^{(\beta)}(A) \times \mathcal{G} \mathcal{M}_{\varphi,\left\{\widehat{\rho}_{n}\right\}, 1 / 4}^{(\beta)}(A),
\end{aligned}
$$

such that $u_{0}=\bar{u}_{0}+\widehat{u}_{0}$ and $u_{1}=\bar{u}_{1}+\widehat{u}_{1}$.

Remark 3.3 Combining Theorem 3.1 and Proposition 3.2 we obtain the following statement: every pair of initial conditions satisfying (2.5) with $r_{0}=\infty$ is the sum of two pairs of initial conditions for which the solution is global. We have thus extended to the general case the astonishing aspect of Manfrin's result.

The extra requirement that $r_{0}=\infty$ is hardly surprising. It is indeed a necessary condition for existence of global solutions even in the theory of linear equations with nonsmooth time dependent coefficients.

Remark 3.4 The $\omega$-continuity assumption on $m$ can be easily relaxed to local $\omega$ continuity in all the cases where there is a uniform-in-time estimate of $\left|A^{1 / 2} u(t)\right|$ in terms of the initial data. We refer to the paragraph "Energy conservation" in section 4.1 for further details.

Remark 3.5 It is possible to extend the result of Theorem 3.1 to larger spaces. A careful inspection of the proof reveals that in the strictly hyperbolic case one can replace $\beta=2$ with any $\beta>1$, in the weakly hyperbolic case one can replace $\beta=3$ with any $\beta>2$. It should also be possible to enlarge these spaces in order to contain all analytic functions, for which a global solution was already known to exist.

Our choice (3.1) is optimized in order to obtain both Theorem 3.1 and Proposition 3.2 under the more general assumptions on $m$, and with a simple proof. 


\section{Proofs}

\subsection{Preliminaries}

Estimates for a continuity modulus The following estimates are crucial in the proof of our main result (see also Lemma 3.1 in [9]).

Lemma 4.1 Let $\omega:[0,+\infty) \rightarrow[0,+\infty)$ be a continuity modulus.

Then

$$
\begin{gathered}
\omega(\lambda x) \leq(1+\lambda) \omega(x) \quad \forall \lambda \geq 0, \quad \forall x \geq 0 ; \\
\omega(x) \geq \omega(1) \frac{x}{x+1} \quad \forall x \geq 0 ; \\
1+\frac{1}{\omega(x)} \leq\left(1+\frac{1}{\omega(1)}\right)\left(1+\frac{1}{x}\right) \quad \forall x>0 .
\end{gathered}
$$

ProOF. Inequality (4.1) can be easily proved by induction on the integer part of $\lambda$ using the monotonicity and the subadditivity of $\omega$. Inequality (4.2) follows from (4.1) applied with $\lambda=1 / x$. Inequality (4.3) follows from (4.2).

Energy conservation Let $u$ be any solution of (1.1) defined in an interval $[0, T)$. Let us set

$$
M(\sigma):=\int_{0}^{\sigma} m(s) d s \quad \forall \sigma \geq 0,
$$

and let us consider the usual Hamiltonian

$$
\mathcal{H}(t):=\left|u^{\prime}(t)\right|^{2}+M\left(\left|A^{1 / 2} u(t)\right|^{2}\right)
$$

It is well known that $\mathcal{H}(t)$ is constant. In particular

$$
\left|u^{\prime}(t)\right|^{2} \leq \mathcal{H}(0) \quad \forall t \in[0, T)
$$

In the strictly hyperbolic case we have also that $M(\sigma) \geq \nu \sigma$, hence

$$
\left|A^{1 / 2} u(t)\right|^{2} \leq \frac{\mathcal{H}(0)}{\nu} \quad \forall t \in[0, T) .
$$

This provides an estimate of $\left|A^{1 / 2} u(t)\right|$ in terms of the initial conditions. This type of estimate can be obtained also without the strict hyperbolicity provided that the limit of $M(\sigma)$ as $\sigma \rightarrow+\infty$ is $+\infty$ or at least larger than $\mathcal{H}(0)$. 
Convolutions In the next result we recall the properties of convolutions which are needed in the sequel (we omit the standard proof).

Lemma 4.2 Let $\rho: \mathbb{R} \rightarrow[0,+\infty)$ be a function of class $C^{\infty}$, with support contained in $[-1,1]$, and integral equal to 1 .

Let $a>0$, and let $f:[0, a] \rightarrow \mathbb{R}$ be a continuous function. Let us extend $f(x)$ to the whole real line by setting $f(x)=f(0)$ for every $x \leq 0$, and $f(x)=f(a)$ for every $x \geq a$.

For every $\varepsilon>0$ let us set

$$
f_{\varepsilon}(x):=\int_{\mathbb{R}} f(x+\varepsilon s) \rho(s) d s \quad \forall x \in \mathbb{R} .
$$

Then $f_{\varepsilon}(x)$ has the following properties.

(1) $f_{\varepsilon} \in C^{\infty}(\mathbb{R})$.

(2) If $\mu_{1} \leq f(x) \leq \mu_{2}$ for every $x \in[0, a]$, then $\mu_{1} \leq f_{\varepsilon}(x) \leq \mu_{2}$ for every $x \in \mathbb{R}$ and every $\varepsilon>0$.

(3) $\left|f_{\varepsilon}(0)\right| \leq \max \{|f(x)|: 0 \leq x \leq \varepsilon\}$ for every $\varepsilon>0$.

(4) Let $\omega$ be a continuity modulus. Let us assume that

$$
|f(x)-f(y)| \leq H \omega(|x-y|) \quad \forall x \in[0, a], \forall y \in[0, a],
$$

for some $H \geq 0$. Then there exists a constant $\gamma_{0}$ (independent on $\varepsilon, H$, and on the function $f(t)$ ) such that

$$
\begin{gathered}
\left|f_{\varepsilon}(x)-f(x)\right| \leq \gamma_{0} H \omega(\varepsilon) \quad \forall x \in \mathbb{R}, \forall \varepsilon>0, \\
\left|f_{\varepsilon}^{\prime}(x)\right| \leq \gamma_{0} H \frac{\omega(\varepsilon)}{\varepsilon} \quad \forall x \in \mathbb{R}, \forall \varepsilon>0 .
\end{gathered}
$$

Maximal local solutions By (3.5) assumptions (3.2) and (3.3) imply that $\left(u_{0}, u_{1}\right) \in$ $\mathcal{G}_{\varphi, \infty, 3 / 4}(A) \times \mathcal{G}_{\varphi, \infty, 1 / 4}(A)$. Therefore the existence of a local solution to $(1.1),(1.2)$ follows from Theorem A both in the strictly hyperbolic and in the weakly hyperbolic case. Since initial data satisfy (2.5) for every $r_{0}$, from the linear theory it easily follows that the local solution satisfies (2.6) for every $r(t)$.

By a standard argument any local solution can be continued to a solution defined in a maximal interval $[0, T)$. If $T=+\infty$ there is nothing to prove. In order to exclude that $T<+\infty$ we prove that the time derivative of $\left|A^{1 / 2} u(t)\right|^{2}$ cannot blow-up in a finite time. The proof of this a priori estimate, which is the basic tool in all global existence results, is different in the strictly hyperbolic and in the weakly hyperbolic case. 


\subsection{The strictly hyperbolic case}

Let us introduce some constants. From the strict hyperbolicity (1.4) and estimate (4.5) we have that

$$
\nu \leq m\left(\left|A^{1 / 2} u(t)\right|^{2}\right) \leq \max \left\{m(\sigma): 0 \leq \sigma \leq \frac{\mathcal{H}(0)}{\nu}\right\}=: \mu \quad \forall t \geq 0 .
$$

Let $L, \Lambda, \gamma_{0}$ be the constants appearing in (2.2), (2.3), and in Lemma 4.2, and let

$$
\begin{gathered}
\gamma_{1}:=\max \{1, \mu\} \cdot \max \left\{1, \nu^{-1}\right\} \\
H_{1}:=\max \left\{\left|\left\langle A^{3 / 4} u_{0}, A^{1 / 4} u_{1}\right\rangle\right|+1,\left(1+\nu^{-1}\right) \mathcal{H}(0)+2 \gamma_{1}+1\right\}, \\
\gamma_{2}:=\gamma_{0} L \Lambda\left(2 H_{1}+1\right)\left(\frac{1}{\nu}+\frac{1}{\sqrt{\nu}}\right) .
\end{gathered}
$$

Since $\rho_{n} \rightarrow+\infty$ we can choose $n \in \mathbb{N}$ such that

$$
\rho_{n} \geq \max \left\{\gamma_{2} T, 1\right\}
$$

Let us set

$$
S:=\sup \left\{\tau \leq T:\left|\left\langle A^{3 / 4} u(t), A^{1 / 4} u^{\prime}(t)\right\rangle\right| \leq H_{1} \rho_{n} \quad \forall t \in[0, \tau]\right\} .
$$

We remark that $S>0$ because $\left|\left\langle A^{3 / 4} u_{0}, A^{1 / 4} u_{1}\right\rangle\right|<H_{1} \leq H_{1} \rho_{n}$.

Now we distinguish the case $S=T$ and $S<T$.

Case $S=T$ The argument is quite standard. In the interval $[0, T)$ the function $u(t)$ is the solution of the linear problem

$$
\begin{gathered}
v^{\prime \prime}(t)+c(t) A v(t)=0 \\
v(0)=u_{0}, \quad v^{\prime}(0)=u_{1},
\end{gathered}
$$

where

$$
c(t):=m\left(\left|A^{1 / 2} u(t)\right|^{2}\right) .
$$

Since $S=T$ in this case we have that

$$
\left.\left|\frac{\mathrm{d}}{\mathrm{d} t}\right| A^{1 / 2} u(t)\right|^{2}|=2|\left\langle A^{3 / 4} u(t), A^{1 / 4} u^{\prime}(t)\right\rangle \mid \leq 2 H_{1} \rho_{n}
$$

for every $t \in[0, T)$. It follows that $\left|A^{1 / 2} u(t)\right|^{2}$ is Lipschitz continuous in $[0, T)$, hence $c(t)$ can be extended to an $\omega$-continuous function defined in the closed interval $[0, T]$. By the linear theory (see [3] and [11]) problem (4.8), (4.9) has a solution

$$
v \in C^{0}\left([0, T] ; \mathcal{G}_{\varphi, r, 3 / 4}(A)\right) \cap C^{1}\left([0, T] ; \mathcal{G}_{\varphi, r, 1 / 4}(A)\right)
$$


for every $r>0$. Since the solution of the linear problem is unique, this implies that there exist

$$
\widehat{u}_{0}:=\lim _{t \rightarrow T^{-}} u(t) \in \mathcal{G}_{\varphi, \infty, 3 / 4}(A), \quad \widehat{u}_{1}:=\lim _{t \rightarrow T^{-}} u^{\prime}(t) \in \mathcal{G}_{\varphi, \infty, 1 / 4}(A) .
$$

Applying Theorem A with initial data $\left(\widehat{u}_{0}, \widehat{u}_{1}\right)$ one can therefore continue $u(t)$ on an interval $\left[0, T_{1}\right)$ with $T_{1}>T$, which contradicts the maximality of $T$.

Case $S<T$ By the maximality of $S$ we have that necessarily

$$
\left|\left\langle A^{3 / 4} u(S), A^{1 / 4} u^{\prime}(S)\right\rangle\right|=H_{1} \rho_{n} .
$$

Let us consider the function $c(t)$ defined according to (4.10). In this case (4.11) holds true for every $t \in[0, S]$, hence by $(2.2)$ and (4.1) we have that

$$
\begin{aligned}
|c(t)-c(s)| & =\left|m\left(\left|A^{1 / 2} u(t)\right|^{2}\right)-m\left(\left|A^{1 / 2} u(s)\right|^{2}\right)\right| \\
& \leq L \omega\left(\left.|| A^{1 / 2} u(t)\right|^{2}-\left|A^{1 / 2} u(s)\right|^{2} \mid\right) \\
& \leq L \omega\left(2 H_{1} \rho_{n}|t-s|\right) \\
& \leq L\left(2 H_{1} \rho_{n}+1\right) \omega(|t-s|) \\
& \leq L\left(2 H_{1}+1\right) \rho_{n} \omega(|t-s|)
\end{aligned}
$$

for every $t$ and $s$ in $[0, S]$. Let us extend $c(t)$ outside the interval $[0, S]$ as in Lemma 4.2, and let us set

$$
c_{\varepsilon}(t):=\int_{\mathbb{R}} c(t+\varepsilon s) \rho(s) d s \quad \forall t \in \mathbb{R} .
$$

Since estimate (4.6) holds true with $H:=L\left(2 H_{1}+1\right) \rho_{n}$, from statements $(2)$ and (4) of Lemma 4.2 we deduce that

$$
\begin{gathered}
\nu \leq c_{\varepsilon}(t) \leq \mu \quad \forall t \in \mathbb{R}, \quad \forall \varepsilon>0, \\
\left|c_{\varepsilon}(t)-c(t)\right| \leq \gamma_{0} L\left(2 H_{1}+1\right) \rho_{n} \omega(\varepsilon) \quad \forall t \in \mathbb{R}, \forall \varepsilon>0, \\
\left|c_{\varepsilon}^{\prime}(t)\right| \leq \gamma_{0} L\left(2 H_{1}+1\right) \rho_{n} \frac{\omega(\varepsilon)}{\varepsilon} \quad \forall t \in \mathbb{R}, \forall \varepsilon>0 .
\end{gathered}
$$

Let us consider the Fourier components $u_{k}(t)$ of $u(t)$, and let us set

$$
E_{k, \varepsilon}(t):=\left|u_{k}^{\prime}(t)\right|^{2}+\lambda_{k}^{2} c_{\varepsilon}(t)\left|u_{k}(t)\right|^{2} \text {. }
$$

An easy computation shows that

$$
\begin{aligned}
E_{k, \varepsilon}^{\prime}(t) & =c_{\varepsilon}^{\prime}(t) \lambda_{k}^{2}\left|u_{k}(t)\right|^{2}+2 \lambda_{k}^{2}\left(c_{\varepsilon}(t)-c(t)\right) u_{k}(t) u_{k}^{\prime}(t) \\
& \leq \frac{\left|c_{\varepsilon}^{\prime}(t)\right|}{c_{\varepsilon}(t)} c_{\varepsilon}(t) \lambda_{k}^{2}\left|u_{k}(t)\right|^{2}+\lambda_{k} \frac{\left|c_{\varepsilon}(t)-c(t)\right|}{\sqrt{c_{\varepsilon}(t)}} 2\left|u_{k}^{\prime}(t)\right| \cdot \lambda_{k} \sqrt{c_{\varepsilon}(t)}\left|u_{k}(t)\right| \\
& \leq \frac{\left|c_{\varepsilon}^{\prime}(t)\right|}{c_{\varepsilon}(t)} E_{k, \varepsilon}(t)+\lambda_{k} \frac{\left|c_{\varepsilon}(t)-c(t)\right|}{\sqrt{c_{\varepsilon}(t)}} E_{k, \varepsilon}(t),
\end{aligned}
$$


hence by (4.14), (4.15), and (4.16) we obtain that

$$
E_{k, \varepsilon}^{\prime}(t) \leq \gamma_{0} L\left(2 H_{1}+1\right) \rho_{n}\left(\frac{1}{\nu} \frac{\omega(\varepsilon)}{\varepsilon}+\frac{1}{\sqrt{\nu}} \lambda_{k} \omega(\varepsilon)\right) E_{k, \varepsilon}(t) \quad \forall t \in[0, S] .
$$

Let us consider now the eigenvalues $\lambda_{k}>\rho_{n}$, which are clearly positive, and let us set $\varepsilon_{k}:=\lambda_{k}^{-1}$. By $(2.3)$ we have that

$$
\frac{\omega\left(\varepsilon_{k}\right)}{\varepsilon_{k}}=\lambda_{k} \omega\left(\varepsilon_{k}\right)=\lambda_{k} \omega\left(\frac{1}{\lambda_{k}}\right) \leq \Lambda \varphi\left(\lambda_{k}\right)
$$

Using these estimates in (4.18) we obtain that

$$
E_{k, \varepsilon_{k}}^{\prime}(t) \leq \gamma_{0} L\left(2 H_{1}+1\right) \rho_{n}\left(\frac{1}{\nu}+\frac{1}{\sqrt{\nu}}\right) \Lambda \varphi\left(\lambda_{k}\right) E_{k, \varepsilon_{k}}(t)=\gamma_{2} \rho_{n} \varphi\left(\lambda_{k}\right) E_{k, \varepsilon_{k}}(t) .
$$

Integrating this differential inequality and using (4.7) we find that

$$
E_{k, \varepsilon_{k}}(t) \leq E_{k, \varepsilon_{k}}(0) \exp \left(\gamma_{2} \rho_{n} \varphi\left(\lambda_{k}\right) T\right) \leq E_{k, \varepsilon_{k}}(0) \exp \left(\rho_{n}^{2} \varphi\left(\lambda_{k}\right)\right)
$$

for every $t \in[0, S]$. Thanks to (4.14) we obtain that

$$
\begin{aligned}
\left|u_{k}^{\prime}(t)\right|^{2}+\lambda_{k}^{2}\left|u_{k}(t)\right|^{2} & \leq \max \left\{1, \nu^{-1}\right\} E_{k, \varepsilon_{k}}(t) \\
& \leq \max \left\{1, \nu^{-1}\right\}\left(\left|u_{1 k}\right|^{2}+\lambda_{k}^{2} c_{\varepsilon_{k}}(0)\left|u_{0 k}\right|^{2}\right) \exp \left(\rho_{n}^{2} \varphi\left(\lambda_{k}\right)\right) \\
& \leq \max \left\{1, \nu^{-1}\right\} \cdot \max \{1, \mu\}\left(\left|u_{1 k}\right|^{2}+\lambda_{k}^{2}\left|u_{0 k}\right|^{2}\right) \exp \left(\rho_{n}^{2} \varphi\left(\lambda_{k}\right)\right) \\
& =\gamma_{1}\left(\left|u_{1 k}\right|^{2}+\lambda_{k}^{2}\left|u_{0 k}\right|^{2}\right) \exp \left(\rho_{n}^{2} \varphi\left(\lambda_{k}\right)\right),
\end{aligned}
$$

where $u_{0 k}$ and $u_{1 k}$ denote the Fourier components of $u_{0}$ and $u_{1}$, respectively.

By assumption (3.2) we have therefore that

$$
\sum_{\lambda_{k}>\rho_{n}} \lambda_{k}\left(\left|u_{k}^{\prime}(t)\right|^{2}+\lambda_{k}^{2}\left|u_{k}(t)\right|^{2}\right) \leq \gamma_{1} \sum_{\lambda_{k}>\rho_{n}} \lambda_{k}\left(\left|u_{1 k}\right|^{2}+\lambda_{k}^{2}\left|u_{0 k}\right|^{2}\right) \exp \left(\rho_{n}^{2} \varphi\left(\lambda_{k}\right)\right) \leq 2 \gamma_{1} \rho_{n}
$$

for every $t \in[0, S]$. On the other hand, by (4.4) and (4.5) we have that

$$
\begin{aligned}
\sum_{\lambda_{k} \leq \rho_{n}} \lambda_{k}\left(\left|u_{k}^{\prime}(t)\right|^{2}+\lambda_{k}^{2}\left|u_{k}(t)\right|^{2}\right) & \leq \rho_{n} \sum_{\lambda_{k} \leq \rho_{n}}\left(\left|u_{k}^{\prime}(t)\right|^{2}+\lambda_{k}^{2}\left|u_{k}(t)\right|^{2}\right) \\
& \leq \rho_{n}\left(\left|u^{\prime}(t)\right|^{2}+\left|A^{1 / 2} u(t)\right|^{2}\right) \\
& \leq \rho_{n}\left(\mathcal{H}(0)+\frac{\mathcal{H}(0)}{\nu}\right)
\end{aligned}
$$


for every $t \in[0, S]$. In particular for $t=S$ we have that

$$
\begin{aligned}
\mid\left\langle A^{3 / 4}\right. & \left.u(S), A^{1 / 4} u^{\prime}(S)\right\rangle\left.|\leq| A^{3 / 4} u(S)\right|^{2}+\left|A^{1 / 4} u^{\prime}(S)\right|^{2} \\
& =\sum_{\lambda_{k} \leq \rho_{n}} \lambda_{k}\left(\left|u_{k}^{\prime}(S)\right|^{2}+\lambda_{k}^{2}\left|u_{k}(S)\right|^{2}\right)+\sum_{\lambda_{k}>\rho_{n}} \lambda_{k}\left(\left|u_{k}^{\prime}(S)\right|^{2}+\lambda_{k}^{2}\left|u_{k}(S)\right|^{2}\right) \\
& \leq \rho_{n}\left(\mathcal{H}(0)+\frac{\mathcal{H}(0)}{\nu}+2 \gamma_{1}\right) \\
& <H_{1} \rho_{n} .
\end{aligned}
$$

This contradicts (4.12).

\subsection{The weakly hyperbolic case}

Let us introduce some constants. Let $L, \Lambda, \gamma_{0}$ be the constants appearing in $(2.2),(2.4)$, and in Lemma 4.2, and let

$$
\begin{gathered}
\gamma_{3}:=1+\frac{1}{\omega(1)}, \\
\gamma_{4}:=\max \left\{m\left(\left|A^{1 / 2} u(t)\right|^{2}\right): t \in[0, T / 2]\right\}+\max \{\omega(\sigma): 0 \leq \sigma \sqrt{\omega(\sigma)} \leq 1\}, \\
\gamma_{5}:=\gamma_{3}\left(1+\gamma_{4}\right)(\Lambda+1) \\
\left.H_{2}:=\max \left\{\left|\left\langle A^{3 / 4} u_{0}, A^{1 / 4} u_{1}\right\rangle\right|+1,\left(\left|u_{0}\right|+1\right) \sqrt{\mathcal{H}(0)}+\gamma_{5}+1\right)\right\}, \\
\gamma_{6}:=1+\gamma_{0} L\left(2 H_{2}+1\right) .
\end{gathered}
$$

Since $\rho_{n} \rightarrow+\infty$ we can choose $n \in \mathbb{N}$ such that $\rho_{n} \geq 1$, and

$$
\rho_{n}^{1 / 2} \geq T \sqrt{\mathcal{H}(0)}, \quad \rho_{n}^{1 / 2} \geq 4 \gamma_{6} \Lambda T, \quad \rho_{n} \geq \frac{2}{T \sqrt{\omega(T / 2)}} .
$$

Let us set

$$
S:=\sup \left\{\tau \leq T:\left|\left\langle A^{3 / 4} u(t), A^{1 / 4} u^{\prime}(t)\right\rangle\right| \leq H_{2} \rho_{n}^{5 / 2} \quad \forall t \in[0, \tau]\right\} .
$$

We remark that $S>0$ because $\left|\left\langle A^{3 / 4} u_{0}, A^{1 / 4} u_{1}\right\rangle\right|<H_{2} \leq H_{2} \rho_{n}^{5 / 2}$.

If $S=T$ we can conclude as in the strictly hyperbolic case (using the linear theory for the weakly hyperbolic case, for which we refer to [4]). So let us assume that $S<T$. By the maximality of $S$ we have that necessarily

$$
\left|\left\langle A^{3 / 4} u(S), A^{1 / 4} u^{\prime}(S)\right\rangle\right|=H_{2} \rho_{n}^{5 / 2} .
$$


Let us consider the function $c(t)$ defined according to (4.10), let us extend it outside the interval $[0, S]$ as in Lemma 4.2 , and let us set

$$
c_{\varepsilon}(t):=\omega(\varepsilon)+\int_{\mathbb{R}} c(t+\varepsilon s) \rho(s) d s \quad \forall t \in \mathbb{R} .
$$

Arguing as in the strictly hyperbolic case we find that

$$
|c(t)-c(s)| \leq L\left(2 H_{2}+1\right) \rho_{n}^{5 / 2} \omega(|t-s|)
$$

for every $t$ and $s$ in $[0, S]$. Therefore from statement (4) of Lemma 4.2 we deduce that

$$
\begin{gathered}
\left|c_{\varepsilon}(t)-c(t)\right| \leq\left(1+\gamma_{0} L\left(2 H_{2}+1\right) \rho_{n}^{5 / 2}\right) \omega(\varepsilon)=\gamma_{6} \rho_{n}^{5 / 2} \omega(\varepsilon), \\
\left|c_{\varepsilon}^{\prime}(t)\right| \leq \gamma_{0} L\left(2 H_{2}+1\right) \rho_{n}^{5 / 2} \frac{\omega(\varepsilon)}{\varepsilon} \leq \gamma_{6} \rho_{n}^{5 / 2} \frac{\omega(\varepsilon)}{\varepsilon} .
\end{gathered}
$$

Let us consider the Fourier components $u_{k}(t)$ of $u(t)$, and let us define $E_{k, \varepsilon}(t)$ as in (4.17). Computing the time derivative as in the strictly hyperbolic case, and using (4.21), (4.22), and the fact that $c_{\varepsilon}(t) \geq \omega(\varepsilon)$ we find that

$$
E_{k, \varepsilon}^{\prime}(t) \leq \gamma_{6} \rho_{n}^{5 / 2}\left(\frac{1}{\varepsilon}+\lambda_{k} \sqrt{\omega(\varepsilon)}\right) E_{k, \varepsilon}(t) \quad \forall t \in[0, S] .
$$

Now we choose $\varepsilon$ as a function of $k$. The function $h(\sigma)=\sigma \sqrt{\omega(\sigma)}$ is invertible. Let us consider the eigenvalues $\lambda_{k}>\rho_{n}$, which are clearly positive, and let us set $\varepsilon_{k}:=h^{-1}\left(1 / \lambda_{k}\right)$. By $(2.4)$ we have that

$$
\lambda_{k} \sqrt{\omega\left(\varepsilon_{k}\right)}=\frac{1}{\varepsilon_{k}} \leq \Lambda \varphi\left(\frac{1}{h\left(\varepsilon_{k}\right)}\right)=\Lambda \varphi\left(\lambda_{k}\right),
$$

hence

$$
E_{k, \varepsilon_{k}}^{\prime}(t) \leq 2 \gamma_{6} \rho_{n}^{5 / 2} \Lambda \varphi\left(\lambda_{k}\right) E_{k, \varepsilon_{k}}(t)
$$

Integrating this differential inequality, and exploiting the second condition in (4.19) we thus obtain that

$$
E_{k, \varepsilon_{k}}(t) \leq E_{k, \varepsilon_{k}}(0) \exp \left(2 \rho_{n}^{5 / 2} \gamma_{6} \Lambda \varphi\left(\lambda_{k}\right) T\right) \leq E_{k, \varepsilon_{k}}(0) \exp \left(\frac{1}{2} \rho_{n}^{3} \varphi\left(\lambda_{k}\right)\right)
$$

for every $t \in[0, S]$. In order to estimate $E_{k, \varepsilon_{k}}(0)$ we need an estimate on $c_{\varepsilon_{k}}(0)$. To this end we first observe that $h\left(\varepsilon_{k}\right)=1 / \lambda_{k}<1$, hence

$$
\omega\left(\varepsilon_{k}\right) \leq \max \{\omega(\sigma): 0 \leq h(\sigma) \leq 1\} .
$$


Moreover the last condition in (4.19) is equivalent to $1 / \rho_{n} \leq h(T / 2)$. Therefore from the monotonicity of $h$ it follows that

$$
\varepsilon_{k}=h^{-1}\left(\frac{1}{\lambda_{k}}\right) \leq h^{-1}\left(\frac{1}{\rho_{n}}\right) \leq h^{-1}\left(h\left(\frac{T}{2}\right)\right)=\frac{T}{2}
$$

hence from statement (3) of Lemma 4.2 we deduce that

$$
\int_{\mathbb{R}} c\left(\varepsilon_{k} s\right) \rho(s) d s \leq \max \left\{c(t): 0 \leq t \leq \varepsilon_{k}\right\} \leq \max \{c(t): 0 \leq t \leq T / 2\} .
$$

From (4.24) and (4.25) it follows that $c_{\varepsilon_{k}}(0) \leq \gamma_{4}$, hence

$$
E_{k, \varepsilon_{k}}(0) \leq \max \left\{1, c_{\varepsilon}(0)\right\}\left(\left|u_{1 k}\right|^{2}+\lambda_{k}^{2}\left|u_{0 k}\right|^{2}\right) \leq\left(1+\gamma_{4}\right)\left(\left|u_{1 k}\right|^{2}+\lambda_{k}^{2}\left|u_{0 k}\right|^{2}\right) .
$$

Moreover from (4.3) and (4.23) it follows that

$$
\max \left\{1, \frac{1}{\omega\left(\varepsilon_{k}\right)}\right\} \leq 1+\frac{1}{\omega\left(\varepsilon_{k}\right)} \leq \gamma_{3}\left(1+\frac{1}{\varepsilon_{k}}\right) \leq \gamma_{3}\left(1+\Lambda \varphi\left(\lambda_{k}\right)\right) .
$$

Since $(1+\Lambda x) \leq(\Lambda+1) e^{x / 2}$ for every $\Lambda \geq 0$ and every $x \geq 0$, we have in particular that

$$
\begin{aligned}
\max \left\{1, \frac{1}{\omega\left(\varepsilon_{k}\right)}\right\} & \leq \gamma_{3}\left(1+\Lambda \varphi\left(\lambda_{k}\right)\right) \leq \gamma_{3}(1+\Lambda) \exp \left(\frac{1}{2} \varphi\left(\lambda_{k}\right)\right) \leq \\
& \leq \gamma_{3}(1+\Lambda) \exp \left(\frac{1}{2} \rho_{n}^{3} \varphi\left(\lambda_{k}\right)\right) .
\end{aligned}
$$

From all these estimates it follows that

$$
\begin{aligned}
\left|u_{k}^{\prime}(t)\right|^{2}+\lambda_{k}^{2}\left|u_{k}(t)\right|^{2} & \leq \max \left\{1, \frac{1}{\omega\left(\varepsilon_{k}\right)}\right\} E_{k, \varepsilon_{k}}(t) \\
& \leq \gamma_{3}(1+\Lambda) E_{k, \varepsilon_{k}}(0) \exp \left(\rho_{n}^{3} \varphi\left(\lambda_{k}\right)\right) \\
& \leq \gamma_{3}(1+\Lambda)\left(1+\gamma_{4}\right)\left(\left|u_{1 k}\right|^{2}+\lambda_{k}^{2}\left|u_{0 k}\right|^{2}\right) \exp \left(\rho_{n}^{3} \varphi\left(\lambda_{k}\right)\right) \\
& =\gamma_{5}\left(\left|u_{1 k}\right|^{2}+\lambda_{k}^{2}\left|u_{0 k}\right|^{2}\right) \exp \left(\rho_{n}^{3} \varphi\left(\lambda_{k}\right)\right) .
\end{aligned}
$$

By assumption (3.3) we have therefore that

$$
\sum_{\lambda_{k}>\rho_{n}} \lambda_{k}\left(\left|u_{k}^{\prime}(t)\right|^{2}+\lambda_{k}^{2}\left|u_{k}(t)\right|^{2}\right) \leq \gamma_{5} \sum_{\lambda_{k}>\rho_{n}} \lambda_{k}\left(\left|u_{1 k}\right|^{2}+\lambda_{k}^{2}\left|u_{0 k}\right|^{2}\right) \exp \left(\rho_{n}^{3} \varphi\left(\lambda_{k}\right)\right) \leq 2 \gamma_{5} \rho_{n}
$$

for every $t \in[0, S]$, and in particular

$$
\begin{aligned}
\left|\sum_{\lambda_{k}>\rho_{n}} \lambda_{k}^{2} u_{k}^{\prime}(S) \cdot u_{k}(S)\right| & \leq \sum_{\lambda_{k}>\rho_{n}} \lambda_{k}^{2}\left|u_{k}^{\prime}(S)\right| \cdot\left|u_{k}(S)\right| \\
& \leq \frac{1}{2} \sum_{\lambda_{k}>\rho_{n}}\left(\lambda_{k}\left|u_{k}^{\prime}(S)\right|^{2}+\lambda_{k}^{3}\left|u_{k}(S)\right|^{2}\right) \\
& \leq \gamma_{5} \rho_{n} .
\end{aligned}
$$


On the other hand, by (4.4) and the first condition in (4.19) we have that

$$
|u(t)| \leq\left|u_{0}\right|+S \cdot \max \left\{\left|u^{\prime}(t)\right|: t \in[0, S]\right\} \leq\left|u_{0}\right|+T \cdot \sqrt{\mathcal{H}(0)} \leq\left(\left|u_{0}\right|+1\right) \rho_{n}^{1 / 2}
$$

for every $t \in[0, S]$, hence

$$
\left|\sum_{\lambda_{k} \leq \rho_{n}} \lambda_{k}^{2} u_{k}^{\prime}(t) u_{k}(t)\right| \leq \rho_{n}^{2}\left|\left\langle u(t), u^{\prime}(t)\right\rangle\right| \leq \rho_{n}^{2}|u(t)| \cdot\left|u^{\prime}(t)\right| \leq \rho_{n}^{5 / 2}\left(\left|u_{0}\right|+1\right) \sqrt{\mathcal{H}(0)}
$$

for every $t \in[0, S]$. In particular for $t=S$ we have that

$$
\begin{aligned}
\left|\left\langle A^{3 / 4} u(S), A^{1 / 4} u^{\prime}(S)\right\rangle\right| & \leq\left|\sum_{\lambda_{k} \leq \rho_{n}} \lambda_{k}^{2} u_{k}^{\prime}(S) \cdot u_{k}(S)\right|+\left|\sum_{\lambda_{k}>\rho_{n}} \lambda_{k}^{2} u_{k}^{\prime}(S) \cdot u_{k}(S)\right| \\
& \leq \rho_{n}^{5 / 2}\left(\left|u_{0}\right|+1\right) \sqrt{\mathcal{H}(0)}+\gamma_{5} \rho_{n} \\
& <H_{2} \rho_{n}^{5 / 2} .
\end{aligned}
$$

This contradicts $(4.20)$.

\subsection{Proof of Proposition 3.2}

Let us recursively define a sequence $\rho_{n}$ as follows. First of all we set $\rho_{0}=0$. Let us assume that a term $\rho_{n}$ has been defined. Assumption (3.6) implies in particular that

$$
\left(u_{0}, u_{1}\right) \in \mathcal{G}_{\varphi, r, 3 / 4}(A) \times \mathcal{G}_{\varphi, r, 1 / 4}(A)
$$

with $r=\rho_{n}^{\beta}$, hence

$$
\sum_{k=1}^{\infty} u_{0 k}^{2} \lambda_{k}^{3} \exp \left(\rho_{n}^{\beta} \varphi\left(\lambda_{k}\right)\right)<+\infty, \quad \sum_{k=1}^{\infty} u_{1 k}^{2} \lambda_{k} \exp \left(\rho_{n}^{\beta} \varphi\left(\lambda_{k}\right)\right)<+\infty .
$$

We can therefore choose $\rho_{n+1}$ big enough in such a way that $\rho_{n+1} \geq \rho_{n}+1$, and

$$
\sum_{\lambda_{k} \geq \rho_{n+1}} u_{0 k}^{2} \lambda_{k}^{3} \exp \left(\rho_{n}^{\beta} \varphi\left(\lambda_{k}\right)\right) \leq \rho_{n}, \quad \sum_{\lambda_{k} \geq \rho_{n+1}}^{\infty} u_{1 k}^{2} \lambda_{k} \exp \left(\rho_{n}^{\beta} \varphi\left(\lambda_{k}\right)\right) \leq \rho_{n} .
$$

Let $\bar{u}_{0}$ and $\bar{u}_{1}$ be the elements of $H$ whose Fourier components are given by

$$
\bar{u}_{0 k}:=\left\{\begin{array}{ll}
0 & \text { if } \rho_{2 k} \leq \lambda_{k}<\rho_{2 k+1}, \\
u_{0 k} & \text { if } \rho_{2 k+1} \leq \lambda_{k}<\rho_{2 k+2},
\end{array} \quad \bar{u}_{1 k}:= \begin{cases}0 & \text { if } \rho_{2 k} \leq \lambda_{k}<\rho_{2 k+1}, \\
u_{1 k} & \text { if } \rho_{2 k+1} \leq \lambda_{k}<\rho_{2 k+2}\end{cases}\right.
$$


and let $\bar{\rho}_{n}:=\rho_{2 n}$. We claim that (3.7) holds true. Indeed for every $n \in \mathbb{N}$ we have that

$$
\begin{aligned}
\sum_{\lambda_{k}>\bar{\rho}_{n}}^{\infty} \bar{u}_{0 k}^{2} \lambda_{k}^{3} \exp \left(\bar{\rho}_{n}^{\beta} \varphi\left(\lambda_{k}\right)\right) & =\sum_{\lambda_{k}>\rho_{2 n}}^{\infty} \bar{u}_{0 k}^{2} \lambda_{k}^{3} \exp \left(\rho_{2 n}^{\beta} \varphi\left(\lambda_{k}\right)\right) \\
& =\sum_{\lambda_{k} \geq \rho_{2 n+1}}^{\infty} \bar{u}_{0 k}^{2} \lambda_{k}^{3} \exp \left(\rho_{2 n}^{\beta} \varphi\left(\lambda_{k}\right)\right) \\
& \leq \sum_{\lambda_{k} \geq \rho_{2 n+1}}^{\infty} u_{0 k}^{2} \lambda_{k}^{3} \exp \left(\rho_{2 n}^{\beta} \varphi\left(\lambda_{k}\right)\right) \\
& \leq \rho_{2 n}=\bar{\rho}_{n},
\end{aligned}
$$

and similarly for $\bar{u}_{1}$. Note that in the second equality we exploited the spectral gap of $\bar{u}_{0}$, whose components are equal to zero in the range $\left(\rho_{2 n}, \rho_{2 n+1}\right)$.

In the same way we can show that $\widehat{u}_{0}:=u_{0}-\bar{u}_{0}$ and $\widehat{u}_{1}:=u_{1}-\bar{u}_{1}$ satisfy (3.8) with $\widehat{\rho}_{n}:=\rho_{2 n+1}$.

\section{References}

[1] A. Arosio, S. Panizzi; On the well-posedness of the Kirchhoff string. Trans. Amer. Math. Soc. 348 (1996), no. 1, 305-330.

[2] A. Arosio, S. Spagnolo; Global solutions to the Cauchy problem for a nonlinear hyperbolic equation. Nonlinear partial differential equations and their applications. Collège de France seminar, Vol. VI (Paris, 1982/1983), 1-26, Res. Notes in Math., 109, Pitman, Boston, MA, 1984.

[3] F. Colombini, E. De Giorgi, S. Spagnolo; Sur les équations hyperboliques avec des coefficients qui ne dépendent que du temp. (French) Ann. Scuola Norm. Sup. Pisa Cl. Sci. (4) 6 (1979), no. 3, 511-559.

[4] F. Colombini, E. Jannelli, S. Spagnolo; Well-posedness in the Gevrey classes of the Cauchy problem for a nonstrictly hyperbolic equation with coefficients depending on time. Ann. Scuola Norm. Sup. Pisa Cl. Sci. (4) 10 (1983), no. 2, 291312.

[5] P. D'Ancona, S. Spagnolo; Global solvability for the degenerate Kirchhoff equation with real analytic data. Invent. Math. 108 (1992), no. 2, 247-262.

[6] P. D'Ancona, S. Spagnolo; On an abstract weakly hyperbolic equation modelling the nonlinear vibrating string. Developments in partial differential equations 
and applications to mathematical physics (Ferrara, 1991), 27-32, Plenum, New York, 1992.

[7] P. D'Ancona, S. Spagnolo; A class of nonlinear hyperbolic problems with global solutions. Arch. Rational Mech. Anal. 124 (1993), no. 3, 201-219.

[8] M. Ghisi, M. GobBino; Derivative loss for Kirchhoff equations with non-Lipschitz nonlinear term. Preprint, arXiv:0805.0244 [math.AP].

[9] M. Ghisi, M. GobBino; A uniqueness result for Kirchhoff equations with nonLipschitz nonlinear term. Preprint, arXiv:0807.1411 [math.AP].

[10] J. M. Greenberg, S. C. Hu; The initial value problem for a stretched string. Quart. Appl. Math. 38 (1980/81), no. 3, 289-311.

[11] H. Hirosawa; Degenerate Kirchhoff equation in ultradifferentiable class. Nonlinear Anal. 48 (2002), no. 1, Ser. A: Theory Methods, 77-94.

[12] H. Hirosawa; Global solvability for Kirchhoff equation in special classes of nonanalytic functions. J. Differential Equations 230 (2006), no. 1, 49-70.

[13] R. MANFRIN; On the global solvability of Kirchhoff equation for non-analytic initial data. J. Differential Equations 211 (2005), no. 1, 38-60.

[14] R. Manfrin; Global solvability to the Kirchhoff equation for a new class of initial data. Port. Math. (N.S.) 59 (2002), no. 1, 91-109.

[15] K. Nishinara; On a global solution of some quasilinear hyperbolic equation. Tokyo J. Math. 7 (1984), no. 2, 437-459.

[16] S. I. Pohozaev; The Kirchhoff quasilinear hyperbolic equation, Differentsial'nye Uravneniya 21 (1985), no. 1 (1985), 101-108 (English transl.: Differential Equations 21 (1985), 82-87). 